\title{
A CLEAR pipeline for direct comparison of circular and linear RNA expression
}

\author{
Xu-Kai Ma', Meng-Ran Wang ${ }^{1}$, Chu-Xiao Liư ${ }^{2}$, Rui Dong ${ }^{1}$, Gordon G. Carmichael ${ }^{3}$, Ling-Ling Chen $^{2,4}$ \\ and Li Yang ${ }^{1,4, *}$
}

${ }^{1}$ CAS Key Laboratory of Computational Biology, CAS-MPG Partner Institute for Computational Biology, Shanghai Institute of Nutrition and Health, Shanghai Institutes for Biological Sciences, University of Chinese Academy of Sciences, Chinese Academy of Sciences, Shanghai 200031, China

${ }^{2}$ State Key Laboratory of Molecular Biology, CAS Center for Excellence in Molecular Cell Science, Shanghai Institute of Biochemistry and Cell Biology, University of Chinese Academy of Sciences, Chinese Academy of Sciences, Shanghai 200031, China

${ }^{3}$ Department of Genetics and Genome Sciences, University of Connecticut Health Center, Farmington, Connecticut 06030, USA

${ }^{4}$ School of Life Science and Technology, ShanghaiTech University, Shanghai 201210, China

* To whom correspondence should be addressed. Tel: 86-21-54920233; Fax: 86-21-54920451; Email: liyang@picb.ac.cn

\section{ABSTRACT}

Sequences of circular RNAs (circRNAs) produced from back-splicing of exon(s) completely overlap with sequences from cognate linear RNAs transcribed from the same gene loci with the exception of their back-splicing junction (BSJ) sites. Examination of global circRNA expression from RNA-seq datasets generally relies on the detection of RNA-seq fragments spanning BSJ sites, but a direct comparison of circular and linear RNA expression from the same gene loci in a genome-wide manner has remained challenging. This is because quantification of BSJ fragments differs from that of linear RNA expression that uses normalized RNA-seq fragments mapped to the whole gene bodies. Here, we have developed a computational pipeline for circular and linear RNA expression analysis from ribosomal-RNA depleted RNA-seq (CLEAR, https://github.com/YangLab/CLEAR). A new quantitation parameter, FPB (ㅁragments per billion mapped bases), is applied to evaluate circular and linear RNA expression individually by fragments mapped to circRNA-specific BSJ sites or to linear RNA-specific splicing junction (SJ) sites. Then, circular and linear RNA expression are directly compared by dividing $\mathrm{FPB}_{\text {circ }}$ by $\mathrm{FPB}_{\text {linear }}$ to generate a CIRCscore, which indicates the relative circRNA expression using linear RNA expression as the background. Highly-expressed circRNAs with low cognate linear RNA expression background can be identified for further investigation.

\section{INTRODUCTION}


Eukaryotic pre-mRNA splicing is catalyzed by spliceosomes to join upstream 5' splice donor sites with downstream 3' splice acceptor sites to produce linear (m)RNAs. Downstream 5' splice donor sites can also be linked to upstream 3' splice acceptor sites, referred to as back-splicing, leading to the production of circular RNAs (circRNAs) from thousands of gene loci (1-3). Unlike most mature linear RNAs (including both coding and long noncoding RNAs), circRNAs lack 3'-end poly (A) tails, resulting in their depletion in poly(A)+ RNA-seq datasets. By taking advantage of analyzing RNA-seq datasets that profile non-polyadenylated transcripts, including ribosomal RNA depletion (ribo-) RNA-seq, and computational approaches that aim to identify fragments mapped to BSJ sites $(4,5)$, a large number of circRNAs have been successfully profiled as being co-expressed with their cognate linear RNAs from the same gene loci $(2,3,6-8)$. Recent research has shown that the biogenesis of circRNAs is catalyzed by canonical spliceosomal machinery and modulated by both cis-elements and trans-factors $(1-3,9,10)$. Importantly, increasing lines of evidence have revealed that circRNAs are involved in physiological and pathological conditions with different modes of actions $(6,11-14)$.

Despite these findings, comprehensive characterization of circRNA biogenesis and function has been impeded by the facts that the majority of circRNAs are processed from middle exons of genes and that their sequences almost completely overlap with those of their cognate linear RNAs except for the BSJ sites (2). No genome-wide method has been available to compare the expression of circRNAs with their cognate linear RNAs directly from RNA-seq datasets. The primary obstacle for direct expression comparison is owing to distinct strategies for circular and linear RNA quantification from mapped RNA-seq fragments. In general, RNA-seq fragments that are solely mapped to BSJ sites are used to represent circRNA expression, such as by raw or normalized (as known as FPM, fragments per million mapped fragments, Figure 1A, left) fragment counts. On the other hand, RNAseq fragments mapped to both exon bodies and exon-exon splicing junction (SJ) sites are summed up and normalized for linear RNA quantification, such as by FPKM (15) (f́ragments per kilobase of transcript per million mapped fragments, Figure 1A, right). Since FPM is unscaled to FPKM, the relative expression of most circRNAs is not comparable to their cognate linear RNAs when analyzing RNA-seq datasets.

To solve this problem, we have developed a computational pipeline for circular and linear RNA expression analysis from ribosomal-RNA depleted RNA-seq (CLEAR, Figure 1B). With the CLEAR pipeline, RNA-seq fragments mapped to circRNA-specific BSJ sites or linear RNA-specific SJ sites are individually normalized to evaluate circular or linear RNA expression, each in FPB (fragments per billion mapped bases). Unlike using the non-comparable FPM and FPKM values, circular and linear RNAs are both quantified by FPB values with the CLEAR pipeline, and thus can be directly compared by dividing $\mathrm{FPB}_{\text {circ }}$ by $\mathrm{FPB}_{\text {linear }}$ to generate a CIRCscore. In this scenario, relative circRNA expression can be evaluated by using linear RNA expression as an expression background, and highlyexpressed circRNAs with low cognate linear RNA expression background can be identified for further functional studies. 


\section{MATERIAL AND METHODS}

\section{Direct circular and linear RNA expression comparison by the CLEAR pipeline}

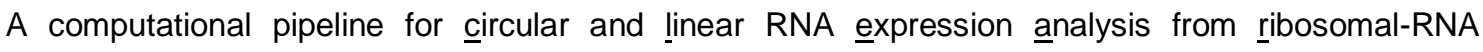
depleted (ribo-) RNA-seq (CLEAR) was developed to achieve direct circular and linear RNA expression comparison. Ribo- RNA-seq datasets that profile both polyadenylated linear and nonpolyadenylated circular RNAs in parallel are used for precise circular and linear RNA expression comparison.

The CLEAR pipeline includes two main steps: alignment and quantification (Figure 1B). For the alignment, ribo- RNA-seq fragments were first mapped by HISAT2 (version 2.0.5; parameters: hisat2 --no-softclip --score-min L,-16,0 --mp 7,7 --rfg 0,7 --rdg 0,7 --dta -k 1 --max-seeds 20) against GRCh38/hg38 human reference genome with known gene annotations (Supplementary Figure S1) for subsequent linear RNA quantification analysis. HISAT2-unmapped fragments were then mapped to the same GRCh38/hg38 reference genome using TopHat-Fusion (version 2.0.12; parameters: tophat2 --fusion-search --keep-fasta-order --bowtie1 --no-coverage-search) for subsequent circRNA quantification.

For the quantification, we developed a new FPB (fragments per billion mapped bases) value to quantitate linear RNA expression by HISAT2-mapped fragments to SJ sites of the maximallyexpressed transcript annotation (Supplementary Figure S2). The maximally-expressed transcript of a given gene is selected with the highest FPKM value, which is calculated by StringTie (version 1.3.3; parameters: stringtie -e -G) from HISAT2 aligned BAM file (16). Fragments mapped to back-splicing junctions were retrieved from TopHat-Fusion as previously reported (version 2.3.6; parameters: CIRCexplorer2 parse - $f$-t TopHat-Fusion) (17) and normalized by totally-mapped bases to obtain FPB values for circRNA quantification.

Direct comparison of circular vs linear RNA expression can be achieved using the CIRCscore value that divides $\mathrm{FBP}_{\text {circ }}$ by $F P B_{\text {linear }}$, which represents relative circRNA expression using linear RNA expression as the background.

\section{Availability and flexibility of the CLEAR pipeline}

The CLEAR pipeline and its application can be downloaded from https://github.com/YangLab/CLEAR. Of note, other aligners, including TopHat2 (version 2.0.12; parameter: tophat2 -a 6 --microexonsearch -m 2 -g 1) with known gene annotations (Supplementary Figure S3) or MapSplice (version 2.1.8 with default parameters) with gene annotations (ensGene_v89.txt updated at 2017/05/08) can also be used in the CLEAR pipeline with similar outputs.

In the CLEAR pipeline, comparable circular or linear RNA expression by FPBs and their direct comparison by the CIRCscore can be obtained directly from raw RNA-seq FASTQ files or processed RNA-seq results, such as CIRCexplorer2 output files (17). Please see https://github.com/YangLab/CLEAR for details.

\section{Cell culture}


PA1 cells were purchased from the American Type Culture Collection (ATCC; http://www.atcc.org), and maintained in MEMa supplemented with $10 \%$ FBS, $1 \%$ Glutamine and $0.1 \%$ penicillin/streptomycin at $37^{\circ} \mathrm{C}$ in a $5 \% \mathrm{CO} 2$ cell culture incubator. PA1 cells were routinely tested to exclude mycoplasma contamination.

\section{Comparison of FPB with qPCR quantification}

Total RNAs from cultured PA1 cells were extracted with Trizol (Life technologies) according to the manufacturer's protocol. Extracted RNAs were treated with DNase I (Ambion, DNA-freeTM kit), reversely transcribed with SuperScript III (Invitrogen) to produce cDNA and then applied for qPCR analysis. $\beta$-actin mRNA was examined as an internal control for normalization. Expression of examined linear and circular RNAs was determined from three independent experiments. The primers used are listed in Supplementary Table S1.

\section{RNA-seq datasets used in this study}

Datasets used for this study include publicly available ribo-, poly $(A)+$, poly $(A)-/$ ribo- and RNase $R$ RNA-seq datasets from PA1 cell line $(17,18)$, ribo- RNA-seq datasets of twelve tissues from ENCODE (19) (Supplementary Table S2), and ribo- RNA-seq datasets of 20 human hepatocellular carcinoma (HCC) samples and their paired control samples (GSE77509) (20).

\section{RESULTS}

\section{Development of the CLEAR pipeline}

The CLEAR pipeline was set up for direct circular and linear RNA expression comparison on a genome-wide scale (Figure 1B). Briefly, ribo- RNA-seq fragments are first mapped to the human reference genome/annotation (NCBI RefSeq genes, Supplementary Figure S1) by HISAT2 (21) to obtain fragments mapped to linear RNAs. Next, HISAT2-unmapped fragments are aligned by TopHatFusion (22) to retrieve fragments mapped to BSJ sites for circRNA quantification (Figure 1B, left, Alignment). Two characteristic features for circular and linear RNA quantification are applied in the CLEAR pipeline. Similar to circRNA quantification by RNA-seq fragments solely mapped to BSJ sites, fragments that only map to canonical splicing site (SJ) sites by HISAT2 are used for linear RNA quantification (Figure 1B, right). Different from commonly-used FPKM that counts fragments mapped to both exon bodies and SJ sites, linear RNA quantification by fragments only mapped to canonical SJ sites is comparable to circRNA quantification by those mapped to BSJ sites (Figure 1B). In addition, fragments mapped to SJ or BSJ sites are normalized by totally mapped bases, rather than by totally mapped fragments, to get FPB for linear or circular RNA quantification (Figure 1B, left). Direct circular and linear RNA expression comparison can be achieved with the CIRCscore that divides $\mathrm{FPB}_{\text {circ }}$ by $\mathrm{FPB}_{\text {linear }}$ (Figure 1B, left).

\section{Comparison of FPB with FPKM for linear RNA quantification}


To evaluate the accuracy of FPB for RNA quantification, commonly-used FPKM values are obtained from the same HISAT2-mapped results. Basically, HISAT2-mapped results are first converted to BAM format by SAMtools (23). StringTie (16) is then used to calculate transcript expression by FPKM. Since multiple linear RNAs can be produced from a given gene locus, the average FPB value of fragments mapped to all SJ sites in the maximally-expressed linear transcript is used to represent the expression of this gene in the current study (Figure 1B and Supplementary Figure S2A, S2B).

With the requirement of $F P B_{\text {linear }}>0$ and $F P K M_{\text {linear }}>0$, linear RNA expression, when quantitated by $\mathrm{FPB}_{\text {linear, }}$, is highly correlated with that by $\mathrm{FPKM}_{\text {linear }}$ in the PA1 cell line (18) (Figure 2A). Indeed, the value of $\mathrm{FPB}_{\text {linear }}$ is theoretically equivalent to that of $\mathrm{FPKM}_{\text {linear }}$ (Supplementary Figure S2C). Furthermore, $\mathrm{FPB}_{\text {linear }}$ is highly correlated with the expression level of thirteen linear RNAs measured by RT-qPCR in PA1 cells (Figure 2B, Supplementary Table S3). We observe a high correlation between $\mathrm{FPB}_{\text {linear }}$ and $\mathrm{FPKM}_{\text {linear }}$ when using different aligners, such as TopHat2 (24) and MapSplice (25), to analyze the ribo- RNA-seq dataset of PA1 (Supplementary Figure S3). Finally, FPB $\mathrm{B}_{\text {linear }}$ is also highly correlated with FPKM linear in ENCODE RNA-seq datasets from twelve examined human tissues (Supplementary Figure S4 and Supplementary Table S2). Collectively, these findings reveal that $\mathrm{FPB}_{\text {linear }}$ is applicable for linear RNA quantification.

\section{Comparison of FPB with FPM for circRNA quantification}

As expected, circRNA expression, when quantitated by $\mathrm{FPB}_{\text {circ }}$, is highly correlated with that by $\mathrm{FPM}_{\text {circ }}$ (Figure $2 \mathrm{C}$ ). Experimentally, $\mathrm{FPB}_{\text {circ }}$ is also highly correlated with the expression of thirteen examined circRNAs measured by RT-qPCR in PA1 cells (Figure 2D, Supplementary Table S3). The expression of these thirteen circRNAs ranges from $\sim 1$ to 10 FPB (Figure 2D), and their cognate linear RNAs are evaluated above (Figure 2B).

Importantly, compared to commonly-used FPM, FPB is resistant to different sequencing lengths and strategies, such as $1 \times 50$ vs $1 \times 100$ or single- vs paired- end RNA-seq datasets (Figure. 2E). These results are in reasonable agreement with the definitions of FPB and FPM. For example, 1 FPB is equivalent to 0.1 FPM for $1 \times 100 \mathrm{bp}$ single-end RNA-seq datasets (Supplementary Figure S5A) and to 0.2 FPM for $2 \times 100$ bp paired-end RNA-seq datasets (Supplementary Figure S5B). In this scenario, FPB can be used directly for cross-sample comparison regardless of different sequencing lengths and strategies.

\section{Evaluation of relative circRNA expression by CIRCscore}

Different from unscaled and non-comparable values of FPKM $_{\text {linear }}$ (for linear RNA expression) and $\mathrm{FPM}_{\text {circ }}$ (for circRNA expression), $\mathrm{FPB}_{\text {linear }}$ for linear RNA measurement is comparable to $\mathrm{FPB}_{\text {circ }}$ for circRNA measurement. We divide $\mathrm{FPB}_{\text {circ }}$ by $\mathrm{FPB}_{\text {linear }}$ to obtain CIRCscore values, by which expression levels of circular and linear RNAs are directly compared in a genome-wide manner. Importantly, the CIRCscore was highly correlated with the experimental comparison of circular vs linear RNA expression measured by RT-qPCR in thirteen examined gene loci from PA1 cells (Figure 2F and Supplementary Table S3), confirming the notion that CIRCscore provides an additional 
parameter to evaluate circRNA expression normalized by their cognate linear RNA expression background.

Since circRNAs are generally co-expressed with their cognate linear RNAs and that sequences of circRNAs largely overlap with those of linear RNAs, the advantage of using CIRCscore to quantitate circRNA expression is that it normalizes their expression to the linear RNA expression background. As shown for in the PA1 cell line, among those with $\mathrm{FPB}_{\text {circ }} \geq 1$, some circRNAs with high FPB values exhibit low CIRCscore values (Figure 3A, blue), which is likely due to the high expression of their cognate linear RNAs (Figure 3B). However, other circRNAs with comparable FPB values have relatively high CIRCscores (Figure 3A, red), as their cognate linear RNAs are expressed at low levels (Figure $3 \mathrm{C}$ ). This observation suggests variable expression patterns of circular and their cognate linear RNAs in different genomic loci.

We further applied CLEAR to evaluate circRNAs in twelve additional human tissues with both FPB and CIRCscore values (Figure 4A and Supplementary Table S4). Consistent with previous findings (26), circRNAs are more abundant in brain samples than in non-brain tissues. Among all six examined brain samples, circRNAs are more enriched in the cortex, occipital and diencephalon, but less in the cerebellum, when evaluated by both FPB (Figure 4A, left) and CIRCscore (Figure 4B, right) values. In six non-brain tissues, circRNAs are enriched in the heart and thyroid at a comparable level as in the cerebellum. About $10 \%-20 \%$ of circRNAs with $\mathrm{FPB}_{\text {circ }} \geq 1$ are expressed at a comparable or even higher level than their cognate linear RNAs, indicated by CIRCscore $\geq 1$ (Figure 4A, right), such as in gene loci for circTPTE2P5 and circPHF7 (Figure 4B). Taken together, the identification of highlyexpressed circRNAs with high $\mathrm{FPB}_{\text {circ }}$ and CIRCscore values reveals that some gene loci are particularly favorable for circRNA production (Supplementary Table S4), and such circRNAs are candidates for subsequent functional studies.

\section{CIRCscore reduces individual differences}

Different to FPB, using CIRCscore to evaluate circRNA expression can reduce individual differences that are caused by RNA-seq samples themselves. For example, compared to paired control samples, circRNA expression evaluated by the $\mathrm{FPB}_{\text {circ }}$ value is inconsistent in a batch of 20 human hepatocellular carcinoma (HCC) samples (GSE77509) (20). Some HCC samples appear to have generally low circRNA expression; while others, such as samples \#11 and \#16, appear to have significantly high circRNA expression (Supplementary Figure S6A). Consequently, it is hard to distinguish circRNA expression differences between HCC and their paired control samples using $\mathrm{FPB}_{\text {circ }}$ in these samples (Figure $5 \mathrm{~A}, P=0.99$ ). Strikingly, it is clearly shown that circRNAs are generally lowly expressed in almost all HCC samples when CIRCscore is used to normalize circRNA expression with cognate linear RNA background (Figure 5B, $P=3.59 \times 10^{-5}$ and Supplementary Figure S6B). These results suggest that it is important to take cognate linear RNA expression into consideration for circRNA quantification, which can be achieved by the CLEAR pipeline in a genomewide manner. Taken together, quantification of circRNA expression by CIRCscore helps to eliminate individual differences among paired comparisons, and it can therefore be used to decipher the trend of circRNA expression changes during different conditions and diseases across RNA-seq datasets. 


\section{DISCUSSION}

Recently, circRNAs have been widely detected in examined cell lines and tissues by deep sequencing of non-polyadenylated RNAs together with specific computational pipelines for detecting RNA-seq reads/fragments mapped to BSJ sites $(17,26,27)$. Due to distinct strategies for circular or linear RNA quantification (Figure 1A), computational pipelines for direct circular and linear RNA expression comparison from RNA-seq datasets have until now been unavailable. In this study, we have developed CLEAR by applying normalized RNA-seq fragments solely mapped to BSJ or canonical SJ sites individually for circular ( $\mathrm{FPB}_{\text {circ }}$ ) or cognate linear $\left(\mathrm{FPB}_{\text {linear }}\right) \mathrm{RNA}$ quantification (Figure 1B).

The CLEAR pipeline has at least two advantages in circRNA studies. First, the FPB values are highly correlated with canonical FPKMs for linear RNAs and FPMs for circRNAs (Figure 2), and unlikely affected by RNA-seq strategies, which makes cross-sample comparisons feasible. Second, direct comparison of circular and cognate linear RNAs with the CIRCscore not only precisely quantitates relative circRNA expression normalized by linear RNA expression background (Figure 3 and 4), but also eliminates possible errors/fluctuations caused by sample preparation/sequencing differences (Figure 5), which reduces inaccuracies for circRNA quantification and subsequent crosssample comparison. Thus, CLEAR has the potential to allow one to identify highly expressed circRNAs in different biological settings for subsequent functional studies. This is important, because so far individual circRNA functions remain to be explored as it is hard even to identify those with high expression levels in the context of interest.

It is worthwhile noting that different RNA sequencing strategies have been applied to profile circRNAs, including ribo-, poly(A)-/ribo- and RNase R-treated RNA-seq datasets (Figure 3). Different from poly $(A)+$ RNA-seq datasets that are used to detect polyadenylated cognate linear RNAs, all three types of non-polyadenylated RNA-seq can be used to determine circRNA expression by FPB. However, only ribo- RNA-seq datasets that profile both polyadenylated linear and non-polyadenylated circular RNAs in parallel are suitable for direct circular and linear RNA expression comparison by CIRCscore (Figure 3). In contrast, in poly(A)-/ribo- and RNase R-treated RNA-seq datasets, polyadenylated linear RNAs are largely depleted, which is unsuitable for accurate linear RNA quantification and subsequent CIRCscore evaluation.

Taken together, the CLEAR pipeline provides a comprehensive way to quantitatively evaluate circRNA expression across samples and to identify highly expressed circRNAs with low linear RNA expression background.

\section{AVAILABILITY}

The CLEAR pipeline and its application can be downloaded from https://github.com/YangLab/CLEAR.

\section{SUPPLEMENTARY DATA}


Supplementary Data are available online.

\section{ACKNOWLEDGEMENT}

We like to acknowledge lab members for testing the CLEAR toolkit.

\section{FUNDING}

This work was supported by the Chinese Academy of Sciences [XDB19020104], the National Natural Science Foundation of China [31730111, 31725009 and 31821004], and the Howard Hughes Medical Institute International Program [55008728].

\section{CONFLICT OF INTEREST}

None declared.

\section{REFERENCES}

1. Chen, L.L. (2016) The biogenesis and emerging roles of circular RNAs. Nat. Rev. Mol. Cell Biol., 17, 205-211.

2. Li, X., Yang, L. and Chen, L.L. (2018) The Biogenesis, Functions, and Challenges of Circular RNAs. Mol. Cell, 71, 428-442.

3. Wilusz, J.E. (2018) A 360 degrees view of circular RNAs: From biogenesis to functions. Wiley Interdiscip Rev RNA, 9, e1478.

4. Hansen, T.B., Veno, M.T., Damgaard, C.K. and Kjems, J. (2016) Comparison of circular RNA prediction tools. Nucleic Acids Res., 44, e58.

5. Gao, Y. and Zhao, F. (2018) Computational Strategies for Exploring Circular RNAs. Trends Genet., 34, 389-400.

6. Memczak, S., Jens, M., Elefsinioti, A., Torti, F., Krueger, J., Rybak, A., Maier, L., Mackowiak, S.D., Gregersen, L.H., Munschauer, M. et al. (2013) Circular RNAs are a large class of animal RNAs with regulatory potency. Nature, 495, 333-338.

7. Zhang, X.O., Wang, H.B., Zhang, Y., Lu, X., Chen, L.L. and Yang, L. (2014) Complementary sequence-mediated exon circularization. Cell, 159, 134-147.

8. Guo, J.U., Agarwal, V., Guo, H. and Bartel, D.P. (2014) Expanded identification and characterization of mammalian circular RNAs. Genome Biol, 15, 409.

9. Jeck, W.R., Sorrentino, J.A., Wang, K., Slevin, M.K., Burd, C.E., Liu, J., Marzluff, W.F. and Sharpless, N.E. (2013) Circular RNAs are abundant, conserved, and associated with ALU repeats. $R N A, 19,141-157$.

10. Liang, D. and Wilusz, J.E. (2014) Short intronic repeat sequences facilitate circular RNA production. Genes Dev., 28, 2233-2247.

11. Hansen, T.B., Jensen, T.I., Clausen, B.H., Bramsen, J.B., Finsen, B., Damgaard, C.K. and Kjems, J. (2013) Natural RNA circles function as efficient microRNA sponges. Nature, 495, 384-388. 
12. Piwecka, M., Glazar, P., Hernandez-Miranda, L.R., Memczak, S., Wolf, S.A., Rybak-Wolf, A., Filipchyk, A., Klironomos, F., Cerda Jara, C.A., Fenske, P. et al. (2017) Loss of a mammalian circular RNA locus causes miRNA deregulation and affects brain function. Science, 357.

13. Kleaveland, B., Shi, C.Y., Stefano, J. and Bartel, D.P. (2018) A Network of Noncoding Regulatory RNAs Acts in the Mammalian Brain. Cell, 174, 350-362 e317.

14. Liu, C.X., Li, X., Nan, F., Jiang, S., Gao, X., Guo, S.K., Xue, W., Cui, Y., Dong, K., Ding, H. et al. (2019) Structure and Degradation of Circular RNAs Regulate PKR Activation in Innate Immunity. Cell, 177, 865-880 e821.

15. Trapnell, C., Williams, B.A., Pertea, G., Mortazavi, A., Kwan, G., van Baren, M.J., Salzberg, S.L., Wold, B.J. and Pachter, L. (2010) Transcript assembly and quantification by RNA-Seq reveals unannotated transcripts and isoform switching during cell differentiation. Nat. Biotechnol., 28, 511-515.

16. Pertea, M., Pertea, G.M., Antonescu, C.M., Chang, T.C., Mendell, J.T. and Salzberg, S.L. (2015) StringTie enables improved reconstruction of a transcriptome from RNA-seq reads. Nat. Biotechnol., 33, 290-295.

17. Zhang, X.O., Dong, R., Zhang, Y., Zhang, J.L., Luo, Z., Zhang, J., Chen, L.L. and Yang, L. (2016) Diverse alternative back-splicing and alternative splicing landscape of circular RNAs. Genome Res, 26: 1277-1287.

18. Zhang, Y., Xue, W., Li, X., Zhang, J., Chen, S., Zhang, J.L., Yang, L. and Chen, L.L. (2016) The Biogenesis of Nascent Circular RNAs. Cell Rep, 15, 611-624.

19. Clarke, L., Zheng-Bradley, X., Smith, R., Kulesha, E., Xiao, C., Toneva, I., Vaughan, B., Preuss, D., Leinonen, R., Shumway, M. et al. (2012) The 1000 Genomes Project: data management and community access. Nat. Methods, 9, 459-462.

20. Yang, Y., Chen, L., Gu, J., Zhang, H., Yuan, J., Lian, Q., Lv, G., Wang, S., Wu, Y., Yang, Y.T. et al. (2017) Recurrently deregulated IncRNAs in hepatocellular carcinoma. Nat Commun, 8, 14421.

21. Kim, D., Langmead, B. and Salzberg, S.L. (2015) HISAT: a fast spliced aligner with low memory requirements. Nat. Methods, 12, 357-360.

22. Kim, D. and Salzberg, S.L. (2011) TopHat-Fusion: an algorithm for discovery of novel fusion transcripts. Genome Biol, 12, R72.

23. Li, H., Handsaker, B., Wysoker, A., Fennell, T., Ruan, J., Homer, N., Marth, G., Abecasis, G., Durbin, R. and Genome Project Data Processing, S. (2009) The Sequence Alignment/Map format and SAMtools. Bioinformatics, 25, 2078-2079.

24. Trapnell, C., Pachter, L. and Salzberg, S.L. (2009) TopHat: discovering splice junctions with RNA-Seq. Bioinformatics, 25, 1105-1111.

25. Wang, K., Singh, D., Zeng, Z., Coleman, S.J., Huang, Y., Savich, G.L., He, X., Mieczkowski, P., Grimm, S.A., Perou, C.M. et al. (2010) MapSplice: accurate mapping of RNA-seq reads for splice junction discovery. Nucleic Acids Res., 38, e178.

26. Rybak-Wolf, A., Stottmeister, C., Glazar, P., Jens, M., Pino, N., Giusti, S., Hanan, M., Behm, M., Bartok, O., Ashwal-Fluss, R. et al. (2015) Circular RNAs in the Mammalian Brain Are Highly Abundant, Conserved, and Dynamically Expressed. Mol. Cell, 58, 870-885.

27. Salzman, J., Chen, R.E., Olsen, M.N., Wang, P.L. and Brown, P.O. (2013) Cell-type specific features of circular RNA expression. PLoS Genet., 9, e1003777. 
A

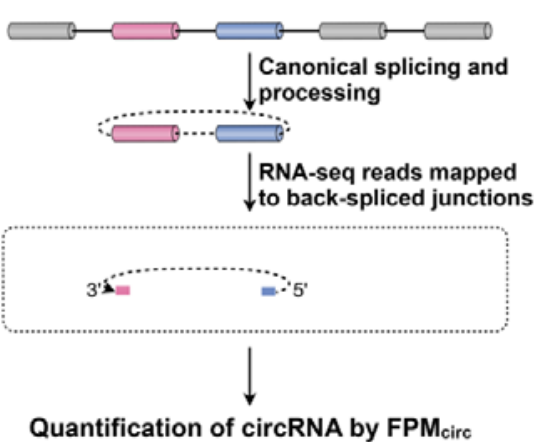

B

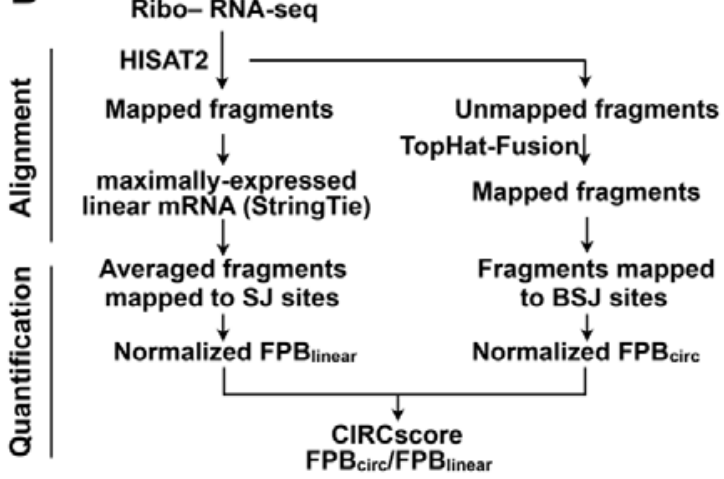

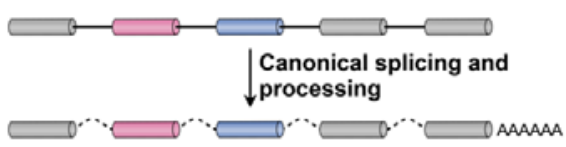

RNA-seq reads mapped to $\downarrow$ exons and exon-exon junctions

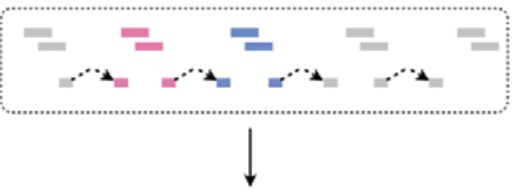

Quantification of linear RNA by FPKM linear

Figure 1. A computational pipeline for direct circular and linear RNA expression comparison. (A) Schematic drawing to show different quantification strategies for circRNAs (left) and linear RNAs (right). Thus, FPM (fragments per million mapped fragments) for circRNA quantification and FPKM (fragments per kilobase of transcript per million mapped fragments) for linear RNA quantification are unscaled and incomparable. (B) Development of the computational pipeline for circular and linear RNA expression analysis from ribosomal-RNA depleted (ribo-) RNA-seq (CLEAR). Schematic diagram of the CLEAR pipeline (left) and the same strategy for circular or linear RNA quantification by splicing junction (SJ) or back-splicing junctions (BSJ) site mapped fragments per billion mapped bases (FPB). CIRCscore that divides $\mathrm{FPB}_{\text {circ }}$ by $\mathrm{FPB}_{\text {linear }}$ can be achieved for direct circular and linear RNA expression comparison. 

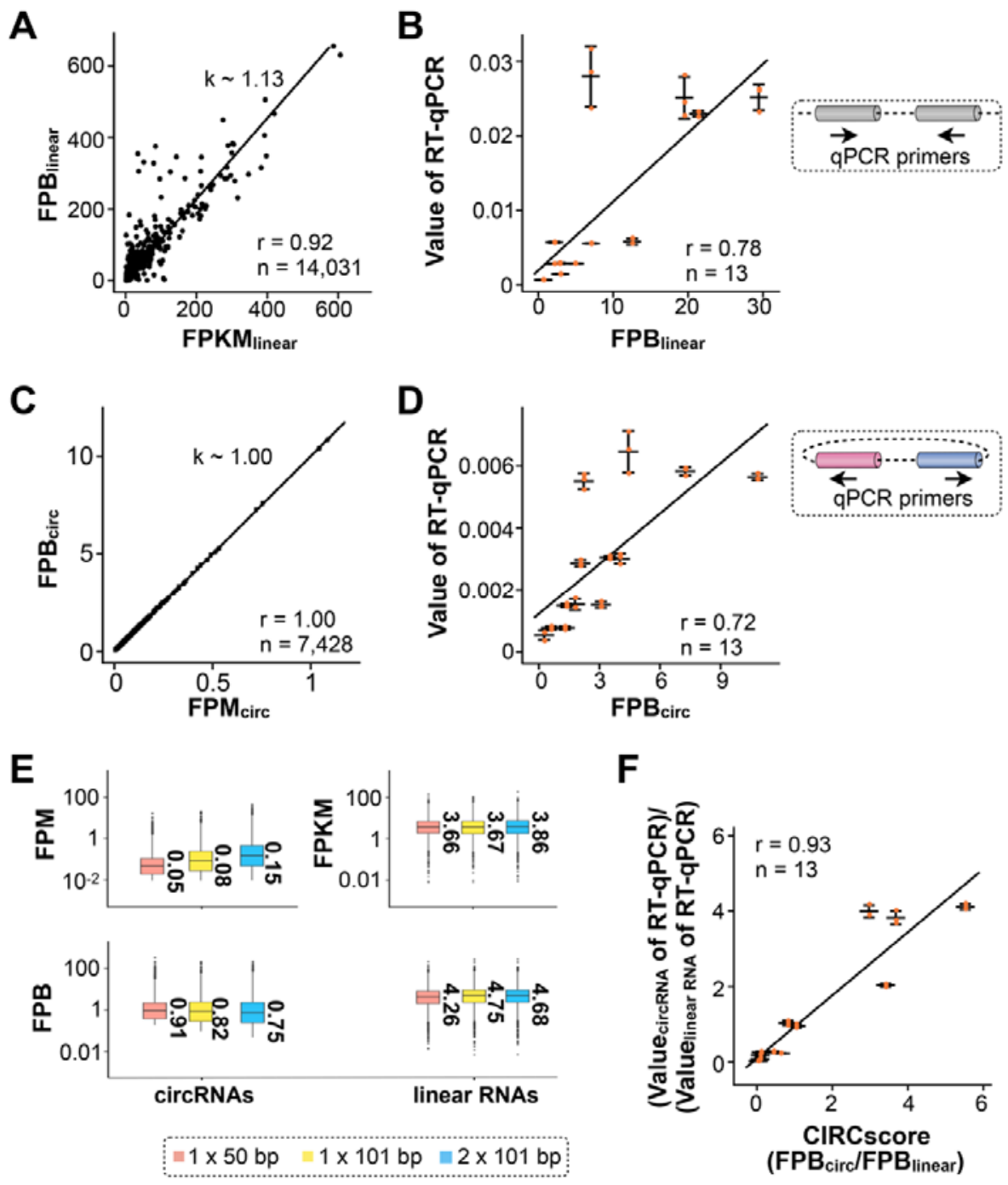

Figure 2. Comparison of FPB with other quantification statistics. (A) Comparison of $\mathrm{FPB}_{\text {linear }}$ and $F P K M_{\text {linear }}$. FPB $B_{\text {linear }}$ is highly correlated with $\mathrm{FPKM}_{\text {linear }}$ (Pearson correlation coefficient $(P C C)=0.92$ ) in PA1 cells, under the condition of both $\mathrm{FPB}_{\text {linear }}$ and $\mathrm{FPKM}_{\text {linear }}>0$. The slope $(\mathrm{k})$ was calculated from linear regression by the $\mathrm{Im}$ function in $\mathrm{R}$. (B) $F P B_{\text {linear }}$ is highly correlated with linear RNA expression measured by RT-qPCR. Expression of thirteen linear RNAs (Supplementary Table S3)

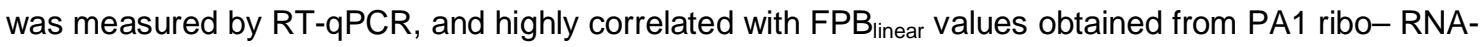
seq $(P C C=0.78)$. Right, qPCR primers are convergently spanned linear RNA exons. Means \pm s.d. were from three independent experiments. (C) Comparison of $\mathrm{FPB}_{\text {circ }}$ and $\mathrm{FPM}_{\text {circ. }} \mathrm{FPB}_{\text {circ }}$ is highly correlated with $F P M_{\text {circ }}(P C C=1.00)$ in PA1 cells. The slope $(k)$ was calculated from linear regression by the Im function in R. (D) $F P B_{\text {circ }}$ is highly correlated with circRNA expression measured by RTqPCR. Expression of thirteen circRNAs (Supplementary Table S3) was measured by RT-qPCR, and highly correlated with $\mathrm{FPB}_{\text {circ }}$ values obtained from PA1 ribo- RNA-seq data (PCC $=0.72$ ). Right, qPCR primers are divergently spanned circRNA exons. Means \pm s.d. were from three independent experiments. (E) FPB is resistant to the changes of sequencing lengths and strategies. Two virtual 
RNA-seq datasets were constructed from original $2 \times 101$ bp cortex ribo- RNA-seq dataset to mimic different sequencing lengths and strategies, including $1 \times 101 \mathrm{bp}$ (extract the left part of reads from the paired-end dataset) and $1 \times 50 \mathrm{bp}$ (extract first $50 \mathrm{bp}$ read sequence from left part of reads). All the three RNA-seq datasets were used for circular and linear RNA quantification to obtain related FPM, FPB and/or FPKM values. Unlike FPM, FPB largely remained unchanged with different sequencing lengths and strategies. Note, FPKM was set as a control that was also not largely altered by the changes of sequencing lengths and strategies. (F) CIRCscore is highly correlated with the value of circular vs linear RNA expression measured by RT-qPCR $(P C C=0.93)$. Means $\pm s . d$. were from three independent experiments. 
A

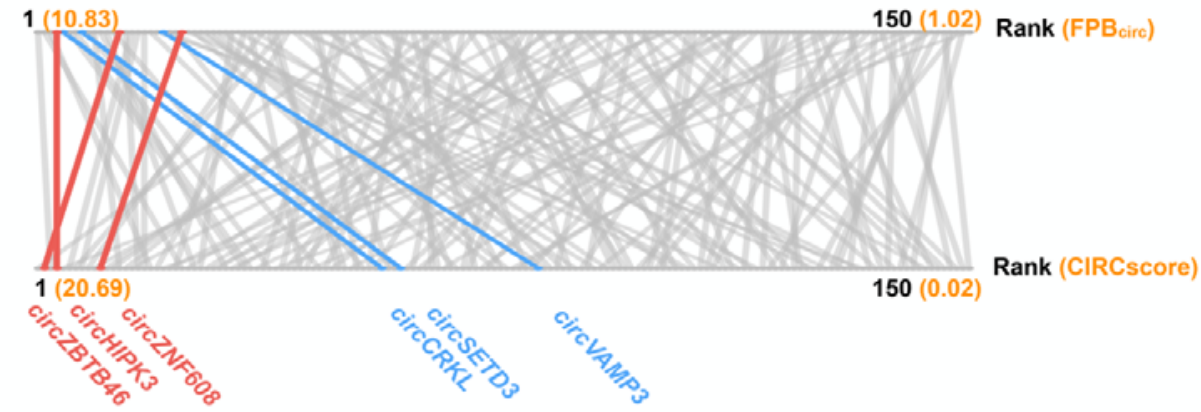

B

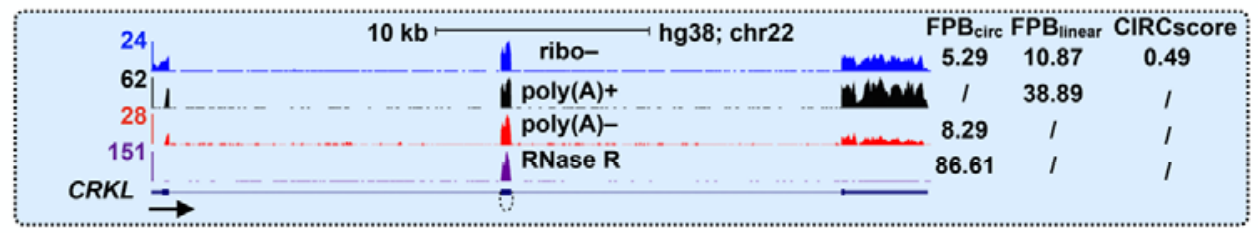

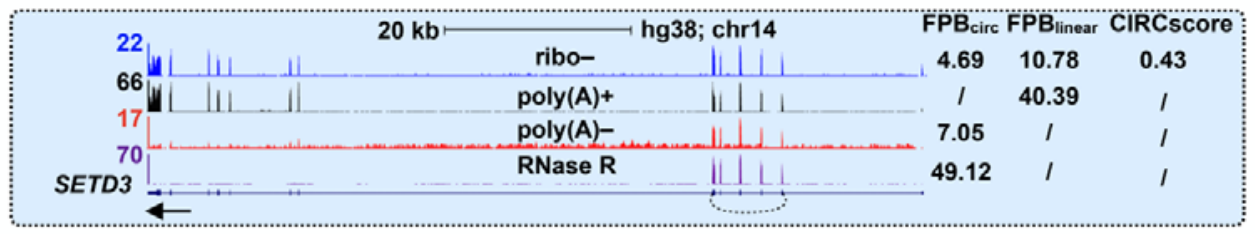

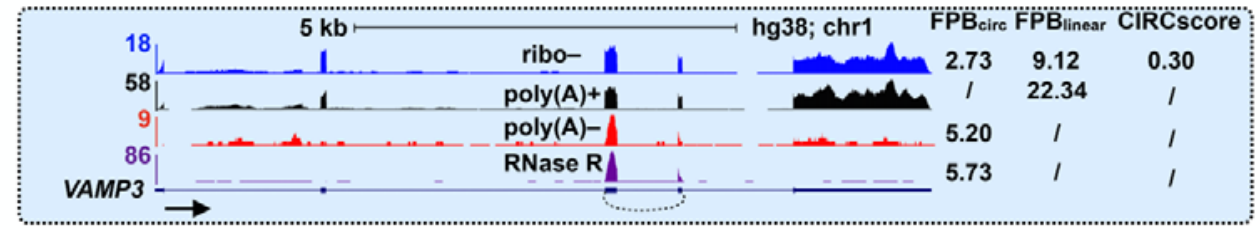

C
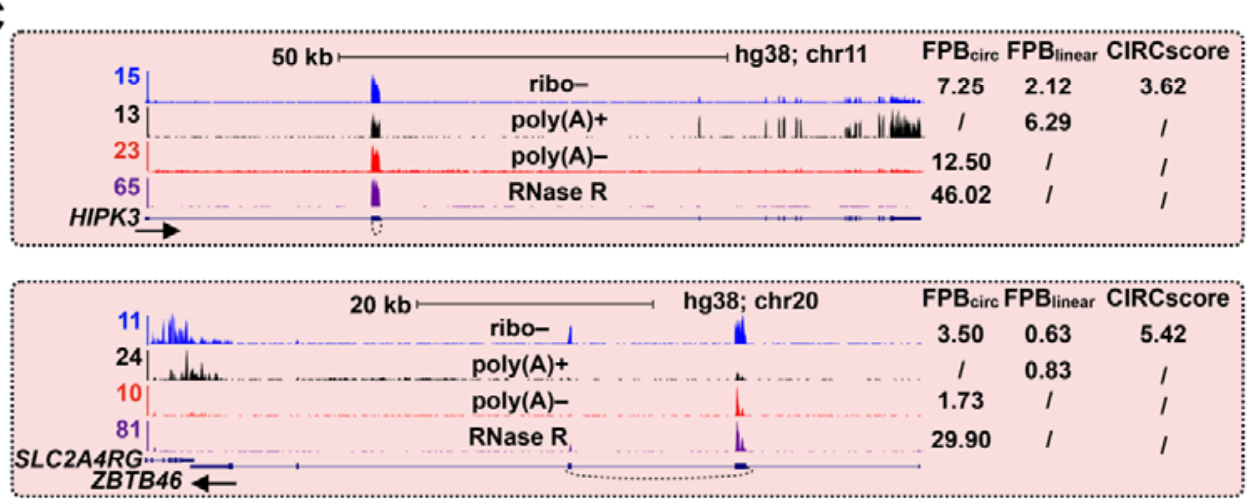

\begin{tabular}{|c|c|c|c|c|c|}
\hline \multirow[b]{2}{*}{9} & \multirow{2}{*}{$50 \mathrm{~kb}$} & \multirow{2}{*}{ hg38; chr5 } & \multicolumn{3}{|c|}{ FPB circ $_{\text {FPB linear }}$ CIRCscore } \\
\hline & & & 2.56 & 1.24 & 2.18 \\
\hline 29 & $\operatorname{poly}(\mathrm{A})+$ & 1 & -1 & 8.89 & 1 \\
\hline 14 & poly(A)- & & 5.07 & 1 & I \\
\hline $\begin{array}{r}118 \\
\text { ZNF608 }\end{array}$ & RNase R & & .76 .70 & I & I \\
\hline
\end{tabular}

Figure 3. Comparison of circRNA quantification by FPB and CIRCscore. (A) Difference of circRNAs quantitated by $\mathrm{FPB}_{\text {circ }}$ or $\mathrm{CIRCscore}$ in PA1 cells. About 150 circRNAs with $\mathrm{FPB}_{\text {circ }} \geq 1$ are identified in 
PA1 cells from published ribo- RNA-seq $(17,18)$. Some circRNAs with high FPB circ $_{\text {values exhibit high }}$ CIRCscore values due to the low background of cognate linear RNA expression (Red), while some others show low CIRCscore values due to the high background of cognate linear RNA expression (Blue). (B) Three highly-expressed circRNAs, circCRKL, circSETD3 and circVAMP3, are coexpressed with their cognate linear RNAs at high levels, indicated by relatively low CIRCscores. (C) Three highly-expressed circRNAs, circHIPK3, circZBTB46 and circZNF608 are co-expressed with their cognate linear RNAs at low levels, indicated by relatively high CIRCscores. 
A

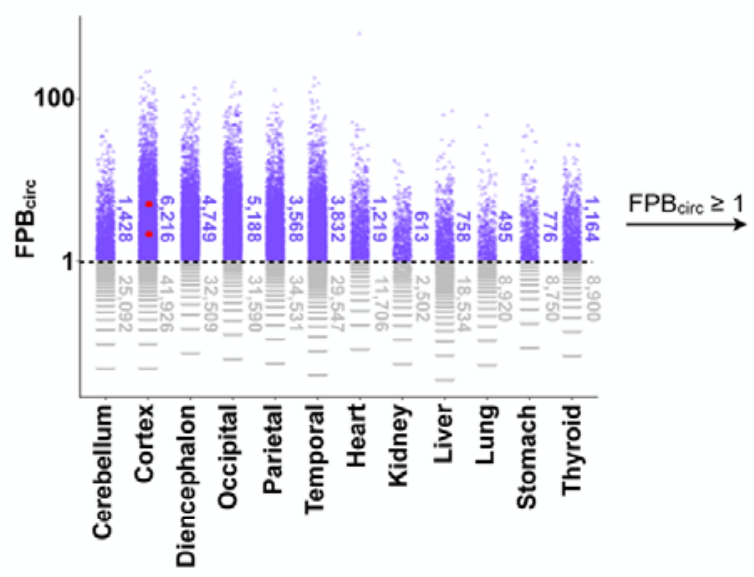

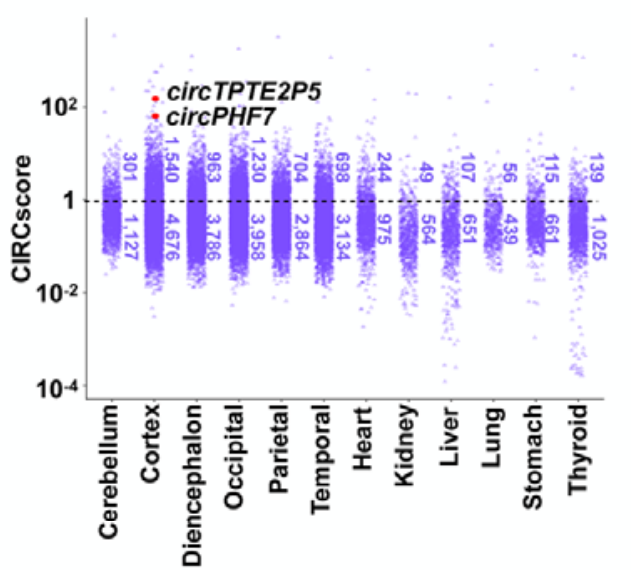

B
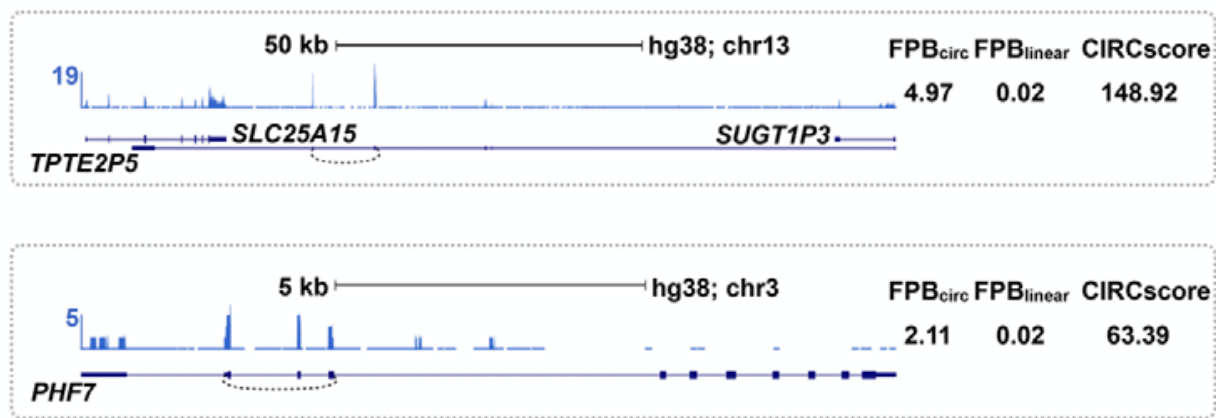

Figure 4. Quantification of circRNAs among twelve human tissue samples. (A) Quantification of circRNAs by the CLEAR pipeline. CircRNAs in twelve ENCODE human tissues are quantitated by $F P B_{\text {circ }}$ (left), and those with $\mathrm{FPB}_{\text {circ }} \geq 1$ (purple) are further evaluated by CIRCscore (Right). Two represented circRNAs in cortex tissue with fair FPB but high CIRCscore values are highlighted in red. (B) Visualization of circTPTE2P5 and circPHF7 in ENCODE human cortex sample. Of Note, circTPTE2P5 and circPHF7 are co-expressed with their cognate linear RNAs at low levels, indicated by high CIRCscores. 
A

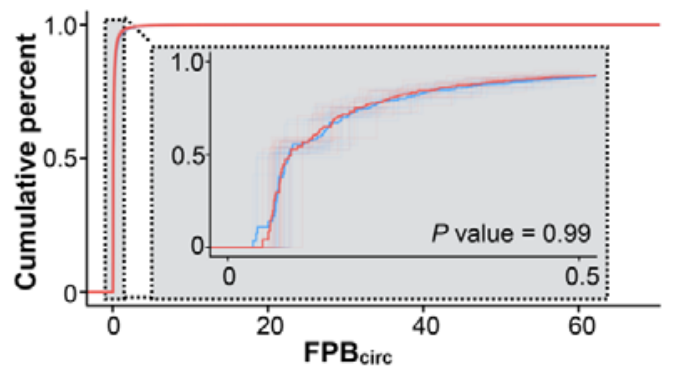

B

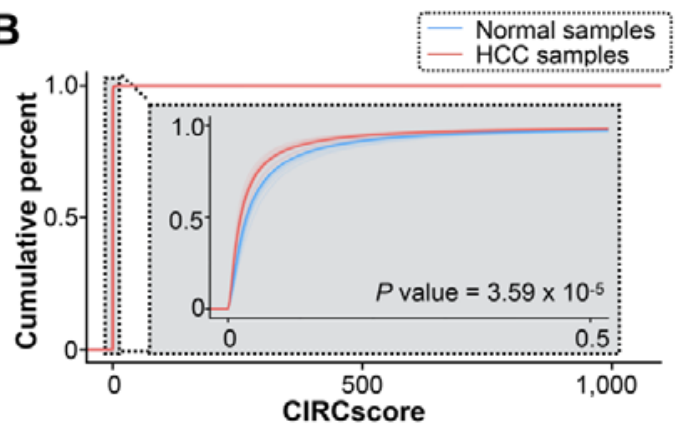

Figure 5. Removal of sample/batch effects with CIRCscore quantification. (A) Cumulative distribution and comparison of circRNAs in 20 paired human hepatocellular carcinoma (HCC) and normal control samples by $\mathrm{FPB}_{\text {circ. }}$. Thick blue and red line represent the mixture distribution of $\mathrm{FPB}_{\text {circ }}$ from 20 normal or HCC samples, respectively. $P$ value for statistical significance of difference between two distributions (normal vs HCC) was calculated using two-tailed unpaired Student's $t$-test. (B) Cumulative distribution and comparison of circRNAs in 20 paired $\mathrm{HCC}$ and normal control samples by CIRCscore. Thick blue and red line represent the mixture distribution of CIRCscores from 20 normal or HCC sample, respectively. $P$ value for statistical significance of difference between two distributions (normal vs HCC) was calculated using two-tailed unpaired Student's t-test. 